\title{
Preoperative Indexed Left Ventricular Dimensions to Predict Early Recovery of Left Ventricular Function After Aortic Valve Replacement for Chronic Aortic Regurgitation
}

\author{
Sang-Ho Cho, MD; Chun-Sung Byun, MD; Kwan-Wook Kim, MD; \\ Byung-Chul Chang, MD, PhD; Kyung-Jong Yoo, MD, PhD; Sak Lee, MD, PhD
}

\begin{abstract}
Background: Aortic valve replacement (AVR) improves left ventricular (LV) systolic function in patients with chronic aortic regurgitation (AR). The objective of this study is to determine predictors for normalization of impaired LV systolic function after valve replacement for chronic AR.
\end{abstract}

\begin{abstract}
Methods and Results: Between 1997 and 2007, 171 patients underwent AVR for severe chronic AR. Of these patients, 79 patients with LV systolic dysfunction or severe LV dilatation preoperatively, who were evaluated by echocardiography at predischarge and early follow up (mean, 6 months) were examined. The mean preoperative ejection fraction was $49 \%$. The mean LV end-systolic and end-diastolic dimensions were $52.32 \pm 8.35 \mathrm{~mm}$ and $69.59 \pm 7.80 \mathrm{~mm}$, respectively. In the early follow up, 62 of 79 patients exhibited restored normal LV function. LV end-systolic dimension and LV end-diastolic dimension were significantly decreased early after AVR (52.32 \pm $8.35 \mathrm{~mm}$ vs $37.82 \pm 6.88 \mathrm{~mm}$, and $69.59 \pm 7.80 \mathrm{~mm}$ vs $51.55 \pm 6.40 \mathrm{~mm}$, respectively). Operative mortality was $3.7 \%$. Multivariate stepwise regression analysis revealed that preoperative indexed LV end-systolic and end-diastolic dimensions were independent predictors of restored LV systolic function. The sensitivity and specificity in predicting normalization of LV function were $88 \%$ and $92 \%$ for indexed LVESD $<35.32 \mathrm{~mm} / \mathrm{m}^{2}$ and $71 \%$ and $86 \%$ for indexed LVEDD $<44.42 \mathrm{~mm} / \mathrm{m}^{2}$.
\end{abstract}

Conclusions: In patients who received a valve replacement for chronic AR, smaller indexed LV systolic and diastolic dimensions were associated with early restoration of LV systolic function. (Circ J 2010; 74: 2340-2345)

Key Words: Aortic valve replacement; Chronic aortic regurgitation; Ventricular function

A ortic regurgitation (AR) causes both volume overload and pressure overload. ${ }^{1}$ As the disease progresses, preload reserve and compensatory hypertrophy permit the ventricle to maintain normal ejection performance with an increase in dimension and wall thickness despite the elevated afterload (called afterload mismatch). Most of these patients are asymptomatic and have an excellent prognosis. ${ }^{2}$

In many patients, however, the balance between afterload excess, preload reserve, and hypertrophy cannot be maintained indefinitely; afterload mismatch, depressed contractility, or severe dilated left ventricular (LV) dimension ultimately results in reduced ejection fraction (EF). ${ }^{2-4}$ The standard of care is to monitor patients with chronic severe AR carefully and operate at the first sign of symptoms or LV dysfunction. ${ }^{1,2,5}$ At this stage, surgical correction of AR can normalize or markedly improve LV systolic function.

Although the hemodynamic rationale for its correction can readily be appreciated in patients with a significantly dilated ventricle and impaired systolic function, surgery sometimes results in persistent LV dilatation and systolic dysfunction. ${ }^{6-9}$ The physiologic changes in the left ventricle after valve replacement are incompletely understood, ${ }^{6}$ but the resulting systolic functional disturbances have important implications for morbidity and mortality before and after aortic valve replacement (AVR). ${ }^{1,3,10}$

In the present study, we assessed the postoperative course after AVR. We evaluated several predictors for early recovery of LV function following AVR in patients with isolated, severe AR combined with LV systolic dysfunction.

Received March 30, 2010; revised manuscript received July 1, 2010; accepted July 12, 2010; released online September 29, 2010 Time for primary review: 21 days

Division of Cardiovascular Surgery, Severance Cardiovascular Hospital, Yonsei University College of Medicine, Yonsei University Health System, Seoul, Republic of Korea

Mailing address: Sak Lee, MD, PhD, Division of Cardiovascular Surgery, Severance Cardiovascular Hospital, Yonsei University College of Medicine, Yonsei University Health System, (120-752) 250 Seongsanno, Seodaemun-gu, Seoul, Republic of Korea. E-mail: sak911@yuhs.ac

ISSN-1346-9843 doi:10.1253/circj.CJ-10-0278

All rights are reserved to the Japanese Circulation Society. For permissions, please e-mail: cj@j-circ.or.jp 


\section{Methods}

From 1997 to 2007, 171 patients underwent primary isolated AVR for chronic AR (at least 6 months' duration) at the Severance Cardiovascular Center of Yonsei University Health System in Korea. Patients with acute AR such as aortic dissection and acute endocarditis, combined aortic stenosis, previous aortic valve surgery, concomitant coronary artery bypass graft, previous or associated mitral valve replacement or repair, and congenital disease unrelated to AR were excluded.

Of these 171 patients, the study group consisted of 79 patients with preoperative LV systolic dysfunction or severe LV dilatation, comprising $47 \%$ of our 171 experiences with AVR for chronic AR.

Preoperative LV systolic dysfunction was defined as EF $<50 \%$. Preoperative severe enlarged LV was defined as LV end-diastolic dimension (LVEDD) $\geq 70 \mathrm{~mm}$ and LV end-systolic dimension (LVESD) $\geq 50 \mathrm{~mm}$. In 30 of the 79 patients, EF was $50-60 \%$ but LV dimension was severely enlarged, however, these patients were still included in this study.

Patients included 54 men (68\%) and 25 women (32\%) with a mean age of $49.0 \pm 14.8$ years. The mean body surface area (BSA) was $1.69 \pm 0.20 \mathrm{~m}^{2}$. Twenty patients $(25 \%)$ and 6 patients $(8 \%)$ had class III and IV heart failure, respectively, according to the New York Heart Association (NYHA) functional classification system. The etiologies of AR were congenital anomaly in 20 patients, rheumatic AR in 16, degeneration in 15, annulo-aortic ectasia in 12, and old infective endocarditis in 11. Preoperative variables are shown in Table 1.

All patients had been treated with angiotensin-converting enzyme (ACE) inhibitors and other medications for heart failure prior to the operation. Hypothermic cardiopulmonary bypass and intermittent antegrade direct cold blood cardioplegia were used routinely for the surgical procedure. Sixty-nine of the patients (87\%) received mechanical valves; $70(89 \%)$ patients had isolated AVR; and $9(13 \%)$ patients underwent concomitant replacement of the ascending aorta (Table 1).

Echocardiography was performed within 1 month before surgery, during the immediate postoperative period ( 7 days after AVR), at early follow up (the mean and median of follow-up time are both 6 months), and at late follow-up appointments. Normalization of LV systolic function was defined as an $\mathrm{EF}>50 \%$. Regular follow up was conducted at an outpatient clinic or by an annual telephone call; follow up was terminated at the end of 2007. The study was approved by the Institutional Review Board of Yonsei University College of Medicine.

\section{Echocardiographic Chamber Quantification}

An echocardiographic assessment was made by using commercially available equipment with tissue Doppler echocardiography according to a standard protocol. Interventricular septal wall thickness and posterior wall thickness, and LV internal dimension at both the end-diastole and end-systole were measured using 2D images from the parasternal longaxis acoustic window. LV end-diastolic volume (EDV) and end-systolic volume (ESV) were measured by the biplane method of disks using 2D images from the apical 4- and 2-chamber views. The EF was calculated by the equation: $100 \times($ EDV-ESV)/EDV. The left atrial (LA) diameter was measured at the end of ventricular systole from both the parasternal long-axis and the apical 4-chamber views.

\begin{tabular}{|c|c|c|}
\hline Patient characteristics & $\mathrm{N}=79$ & Mean \pm SD \\
\hline Age (years) & & $49.0 \pm 14.8$ \\
\hline BSA $\left(m^{2}\right)$ & & $1.69 \pm 0.20$ \\
\hline Male & 54 & $69 \%$ \\
\hline \multicolumn{3}{|l|}{ NYHA } \\
\hline I & 20 & $25 \%$ \\
\hline II & 33 & $42 \%$ \\
\hline III & 20 & $25 \%$ \\
\hline IV & 6 & $8 \%$ \\
\hline \multicolumn{3}{|l|}{ Etiology of AR } \\
\hline Congenital & 20 & $25 \%$ \\
\hline Bicuspid & 19 & \\
\hline Quadricuspid & 1 & \\
\hline Rheumatic & 16 & $20 \%$ \\
\hline Degenerative & 15 & $19 \%$ \\
\hline Aneurysm/annular dilatation & 12 & $15 \%$ \\
\hline Old infective endocarditis & 11 & $15 \%$ \\
\hline Aortitis & 5 & $6 \%$ \\
\hline \multicolumn{3}{|l|}{ EF (\%) } \\
\hline$<25$ & 9 & $12 \%$ \\
\hline $25<<50$ & 40 & $50 \%$ \\
\hline $50<<60$ & 30 & $38 \%$ \\
\hline \multicolumn{3}{|l|}{ Procedure } \\
\hline Mechanical & 69 & $87 \%$ \\
\hline Bioprosthesis & 10 & $13 \%$ \\
\hline AVR only & 70 & $88 \%$ \\
\hline $\begin{array}{l}\text { Composite AVR and ascendina aorta } \\
\text { replacement }\end{array}$ & 7 & $9 \%$ \\
\hline $\begin{array}{l}\text { Separate AVR and ascendina aorta } \\
\text { replacement }\end{array}$ & 2 & $3 \%$ \\
\hline
\end{tabular}

BSA, body surface area; NYHA, New York Heart Association; $A R$, aortic regurgitation; $E F$, ejection fraction; AVR, aortic valve replacement.

\section{Statistical Analyses}

Continuous variables are expressed as mean \pm standard deviation. Cumulative survival was estimated by the KaplanMeier method using the date of surgery and date of the most recent follow up. Differences in survival rates were determined by log-rank analysis. The paired t-test was used to compare 2 groups, while ANOVA was used to compare more than 2 groups. A multivariable analysis of independent factors was performed by using stepwise regression. For continuous variables, diagnostic cut-off values with the most favorable sensitivities and specificities were identified by receiveroperating characteristics curve analysis. Statistical significance was defined as a 2-tailed P-value $<0.05$. SPSS for Windows, Release 15.0 (SPSS Inc, Chicago, IL, USA) was used for statistical analysis.

\section{Results}

Among the 171 patients who underwent AVR at our hospital, operative mortality was $2.9 \%(5 / 171)$, and overall survival was $89.8 \%$, whereas operative mortality of the 79 patients in the present study was $3.7 \%$ (3/79), and overall survival was $86.9 \%$. However, there was no significant difference in overall survival (operative and late deaths combined) between the 79 patients of our study group and the patients with preserved 


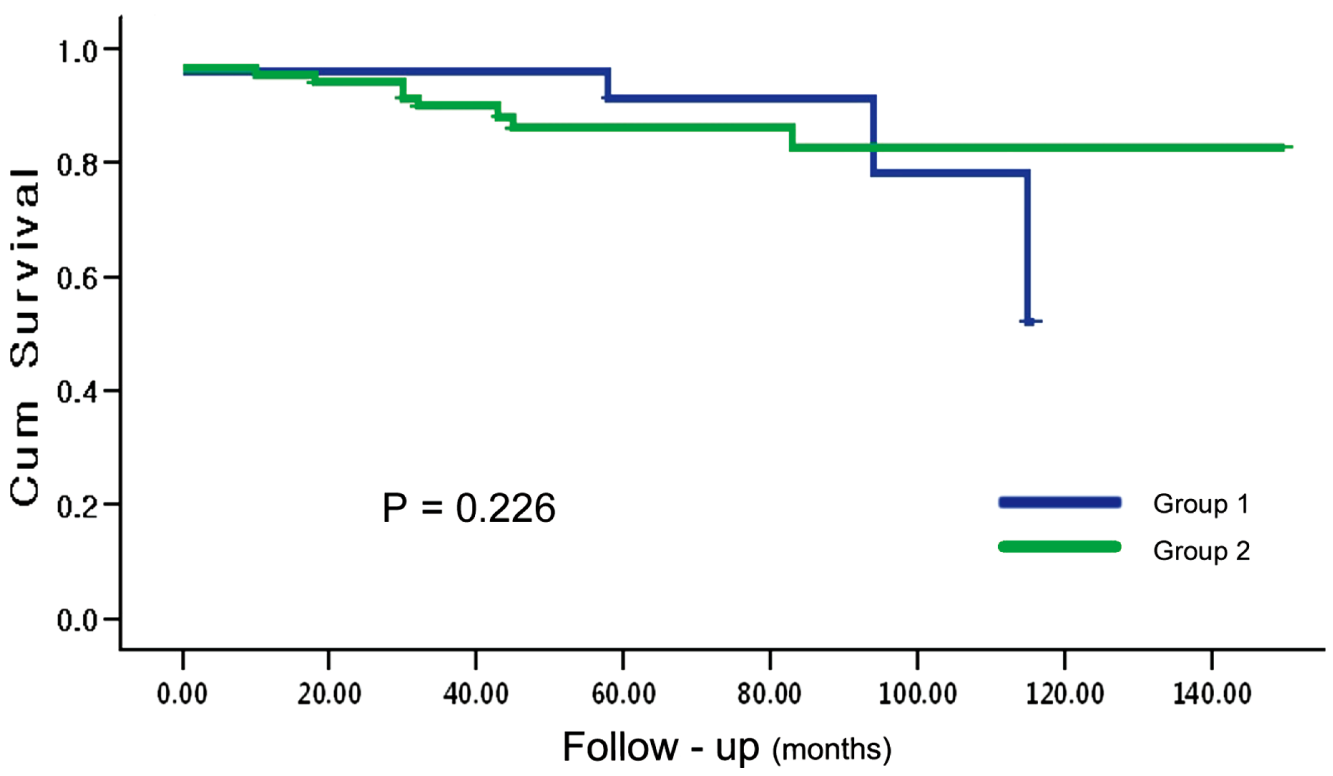

Figure 1. Comparison of cumulative survival at 10 years. Group 1, Patients with preserved left ventricular (LV) function; Group 2 , Patients with decreased LV function.

\begin{tabular}{|c|c|c|c|c|}
\hline & Preoperative & Immediate postoperative & Early postoperative & $P$ value \\
\hline LVEF (\%) & $49 \pm 10$ & $43 \pm 13$ & $55 \pm 11$ & $<0.001$ \\
\hline LVESD $(\mathrm{mm})$ & $52.32 \pm 8.35$ & $44.45 \pm 8.82$ & $37.82 \pm 6.88$ & $<0.001$ \\
\hline Indexed LVESD $\left(\mathrm{mm} / \mathrm{m}^{2}\right)$ & $31.23 \pm 6.10$ & $26.75 \pm 6.38$ & $22.42 \pm 5.78$ & $<0.001$ \\
\hline LVEDD (mm) & $69.59 \pm 7.80$ & $56.44 \pm 7.53$ & $51.55 \pm 6.40$ & $<0.001$ \\
\hline Indexed LVEDD $\left(\mathrm{mm} / \mathrm{m}^{2}\right)$ & $41.47 \pm 6.23$ & $33.40 \pm 5.86$ & $30.80 \pm 4.91$ & $<0.001$ \\
\hline
\end{tabular}

Preoperative, within 1 month before surgery; Immediate postoperative, 7 days after AVR; Early postoperative, 6 months (mean, median) after AVR; LVEF, left ventricular (LV) ejection fraction; LVESD, LV end-systolic dimension; LVEDD, LV end-diastolic dimension. Other abbreviation see in Table 1.

Comparison of the 3 groups was by ANOVA and Tukey's multiple comparison test.

LV function and LV dimension (Figure 1). In a multivariate analysis using the Cox regression model, no variables were found to be independent predictors of operative mortality and overall survival.

Table 2 shows the variables before and after the operation. Before the procedure, the mean preoperative EF was $49 \pm$ $10 \%$, the mean LVESD was $52.32 \pm 8.35 \mathrm{~mm}$, and the mean LVEDD was $69.59 \pm 7.80 \mathrm{~mm}$. In the immediate postoperative period, the mean EF and LV dimensions were decreased. An early postoperative follow-up echocardiography (median, 6 months; mean, $6.43 \pm 2.03$ months) showed that the mean EF increased from 49 to $55 \%$. Both LVESD and LVEDD were significantly decreased after AVR $(52.32 \pm 8.35 \mathrm{~mm}$ vs $37.82 \pm 6.88 \mathrm{~mm}, 69.59 \pm 7.80 \mathrm{~mm}$ vs $51.55 \pm 6.40 \mathrm{~mm}$, respectively). At all time-points, the mean EFs and LV dimensions were significantly different, according to analysis of variance (ANOVA) and Tukey's multiple comparison test. Sixty-two of the 79 patients $(78 \%)$ achieved normal LV systolic function in the early postoperative period. A late follow-up echocardiography (median, 35 months; mean 45 months; range, 12 months to 10 years) showed that LV systolic function was not restored in 7 of the remaining 17 patients $(41 \%)$.

Univariate risk analysis demonstrated that NYHA func- tional classes, preoperative EF, LV dimensions, indexed LV dimensions, and LA volume index were significant risk factors for early recovery of LV function after AVR. The immediate postoperative change in LVEF was not predictive of normal EF during the early preoperative period; however, multivariate stepwise regression analysis showed that preoperative indexed LVESD and preoperative indexed LVEDD were significant predictors (odds ratios [OR], 7.60 and 0.383 , respectively) (Table 3 ).

Using receiver-operating characteristics curves, we found that the sensitivity and specificity for predicting the normalization of EF early after AVR were $86 \%$ and $71 \%$ for indexed LVEDD $\left(<44.42 \mathrm{~mm} / \mathrm{m}^{2}\right), 68 \%$ and $91 \%$ for indexed LVESD $\left(<35.32 \mathrm{~mm} / \mathrm{m}^{2}\right)$ (Figure 2). In other words, patients with an indexed LVEDD $>44.42 \mathrm{~mm} / \mathrm{m}^{2}$ and an indexed LVESD $>35.32 \mathrm{~mm} / \mathrm{m}^{2}$ had only a $29 \%$ and $9 \%$ chance of LVEF normalization after AVR, respectively.

\section{Discussion}

In patients with severe chronic AR, the presence of symptoms is considered a class I indication for AVR. In asymptomatic patients, surgery is recommended when LV dysfunc- 
tion, defined as end-systolic diameter (ESD), is greater than $50-55 \mathrm{~mm}$ and/or the EF is less than $50 \% .^{5}$ These guidelines are based on previous studies reporting that LV dimensions and preoperative systolic function are important predictors of postoperative prognosis. Recent studies have suggested the use of indexed LV dimensions in chronic AR to optimize surgical indications because decisions based on raw data might be inappropriate for patients with extreme body surface area values. ${ }^{11,12}$ Sambola et al showed that indexed ESD (IESD $\geq 25 \mathrm{~mm} / \mathrm{m}^{2}$ ) predicts unfavorable postoperative outcomes more accurately than ESD (ESD >50 mm) in patients with AR. ${ }^{11}$ This effect is more pronounced in patients with a low body surface area. Actually, Asian hearts are small compared with the reference values in the guidelines from the American Society of Echocardiography and BSA is also significantly low. ${ }^{13}$ Other investigators showed that larger indexed LV dimensions were associated with late mortality, indicating the importance of indexed values. ${ }^{12}$ In the present study, we therefore assessed the postoperative course of patients with isolated, severe AR combined with LV systolic dysfunction. We also evaluated several predictors containing indexed values of echocardiographic parameters following AVR.

\section{Mortality and Late Survival}

Earlier studies showed that patients with markedly low EF $(<35 \%)$ or dilated LV constitute a high-risk group even after successful surgery. ${ }^{8,9,12}$ Similarly, Chaliki et al reported that low EF is associated with excessive operative mortality (OR 4.3) and long-term mortality (OR 2.3) compared with normal EF. ${ }^{8}$ Even when excluding operative mortality rates, the risk ratio for expected mortality with low EF was 3.2 compared with 1.4 for normal EF. ${ }^{8}$ A study at the Cleveland Clinic, however, reported no significant difference in operative mortality and long-term survival in patients with LV dysfunction (EF <35\%) who underwent surgery after 1985 compared with patients with preserved LV function. ${ }^{14}$ The declining operative mortality for such patients is most likely due to

\begin{tabular}{|c|c|c|c|}
\hline & Univariate & Multivariate OR & $P$ value \\
\hline Age & 0.440 & & \\
\hline Sex & 0.823 & & \\
\hline NYHA class & 0.001 & & \\
\hline $\mathrm{EF}$ & $<0.001$ & & 0.0642 \\
\hline LVEDD & $<0.001$ & & \\
\hline $\begin{array}{l}\text { Indexed LVEDD } \\
\text { (LVEDD/BSA) }\end{array}$ & $<0.001$ & 0.383 & 0.0456 \\
\hline LVESD & $<0.001$ & & \\
\hline $\begin{array}{l}\text { Indexed LVESD } \\
\text { (LVESD/BSA) }\end{array}$ & $<0.001$ & 7.60 & 0.0095 \\
\hline Changes in LVEDD* & 0.561 & & \\
\hline Changes in LVESD* & 0.669 & & \\
\hline Changes in $\mathrm{EF}^{\star}$ & 0.792 & & \\
\hline IVSd & 0.372 & & \\
\hline IVSs & 0.568 & & \\
\hline PWd & 0.463 & & \\
\hline PWs & 0.875 & & \\
\hline LA volume & 0.010 & & \\
\hline LV mass & 0.400 & & \\
\hline RVP & 0.265 & & \\
\hline
\end{tabular}

OR, odds ratios; EF, ejection fraction; IVSd, diastolic interventricular septal thickness; IVSs, systolic interventricular septal thickness $\mathrm{PWd}$, diastolic posterior wall thickness; PWs, systolic posterior wall thickness; LA, left atrial; RVP, right ventricular pressure. Other abbreviations see in Tables 1,2.

${ }^{*}$ Changes in, Difference between immediate postoperative and preoperative echocardiograph.

multifactorial causes, such as improvements in surgical and anesthetic techniques, early surgery, cardioprotection, prosthetic valve design, postoperative care, and concomitant medical therapy. This result is consistent with the present study, in which the operative mortality was $3.7 \%$ for patients

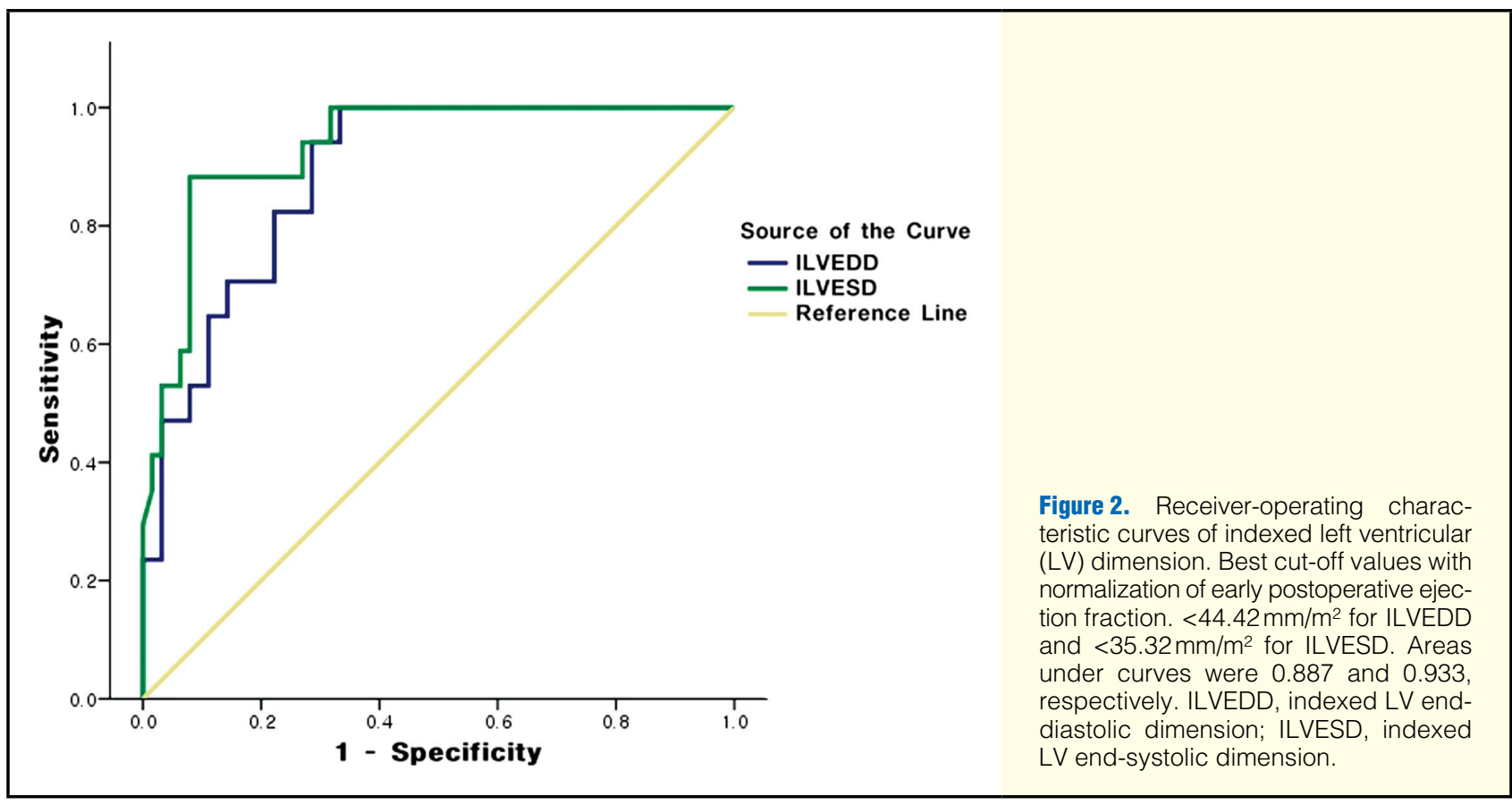


with LV dysfunction. Regarding overall survival (operative and late deaths combined), there was no significant difference between the 79 patients of our study group and patients with preserved LV function and LV dimension. We were unable to identify predictors for mortality and late survival. Brown et al showed that decreased LVEF and increased LV dimensions were not associated with late mortality, but that larger indexed LV systolic and diastolic dimensions predicted late mortality. ${ }^{12}$ Klodas et al showed that extreme LV dilatation was not predictive of mortality and overall survival, and that preoperative low EF was an independent predictor of survival. ${ }^{9}$

\section{Improvement in LV Systolic Function After AVR}

Enlarged LV dimension is usually reduced within the first few weeks after correction of AR, but EF also decreases at approximately the same time because the effect of preload change is likely to be dominant at this time..$^{915-17}$ However, the EF eventually returns to or surpasses preoperative values. This change might be a result of the reduced afterload with gradual improvement in LV function, possibly related to regression of LV hypertrophy after AVR, or it might represent an actual improvement in contractile function. ${ }^{8,9,15,16,18}$ In patients with a significantly dilated ventricle and impaired systolic function, surgery sometimes results in persistent LV dilatation and LV systolic dysfunction. ${ }^{6,9,17,19}$ Because LV functional recovery after AVR is associated with relief of symptoms and prognosis in patients with chronic $\mathrm{AR}$, it is useful to know preoperative predictors for LV functional recovery after AVR.1,9,12 Several studies have demonstrated that the early postoperative status substantially influences long-term outcomes. Thus, patients who have an increase in LVEF within a few months after AVR for AR can expect further improvement in ventricular function. ${ }^{6,9,16}$ Fewer studies, however, have focused on the effect of preoperative status on early recovery of LV function. Previous investigators reported that preoperative EF was the only significant independent predictor of late recovery of LV function., ${ }^{9,12}$ In contrast, our present study evaluated the early postoperative course in patients with preexisting $\mathrm{LV}$ dysfunction. The mean EF was increased from $49 \%$ to $55 \%$ at early follow up, and LVESD and LVEDD were significantly decreased (52.32 $8.35 \mathrm{~mm}$ vs $37.82 \pm 6.88 \mathrm{~mm}$ and $69.59 \pm 7.80 \mathrm{~mm}$ vs $51.55 \pm$ $6.40 \mathrm{~mm}$, respectively). Despite the correction of severe regurgitation, LV function was not restored in $22 \%$ of the patients; this result was associated with large preoperative indexed LV dimensions. Although our findings were not statistically significant $(\mathrm{P}=0.06)$, we could not rule out preoperative $\mathrm{EF}$ as a predictor of early outcomes. Therefore, this issue for the preoperative EF requires further evaluation.

\section{Clinical Implication}

In chronic isolated AR, AVR should ideally be performed before severe EF decrease and LV dilatation. Previous studies demonstrated that persistent ventricular dilatation and dysfunction identifies patients at risk of death from congestive heart failure. ${ }^{17,19}$ Recently, operative mortality and overall survival was shown to decline after AVR in a high-risk group. Therefore, we prefer surgical correction over medically conservative care even in high-risk patients. On the basis of our findings and those of other investigators, early surgical treatment might be important for postoperative clinical outcomes even in patients with severe LV dilatation and LV dysfunction, considered with reference to indexed LV dimensions. In the present study, the cut-off values for indexed
LV dimensions were not indicators of surgical correction, but indicated the upper limit for delaying AVR.

\section{Study Limitations}

There are several limitations in this study. First, this study is a retrospective study. In addition, we did not evaluate preoperative predictors for mid-term or long-term LV function because mid-term or late follow-up echocardiograms were obtained in only 50 of the 79 patients. However, previous studies demonstrated that early postoperative recovery of LV function was independently associated with long-term outcome and relief of symptoms. Second, postoperative NYHA functional class changes were not available for all patients; therefore, we were unable to determine the relationship between recovery of LV function and improvement of heart failure symptoms. Finally, our study group was made up of patients with LV dysfunction. Preoperative LV systolic dysfunction is generally defined as $\mathrm{EF}<50 \%$; however, in the present study, EF was $50-60 \%$ in 30 of the 79 patients, but LV dimension was enlarged and global LV hypokinesia was detected by echocardiography. In AR, an abnormally increased preload by large regurgitant volume and an altered afterload might result in a overestimated EF despite underlying myocardial dysfunction (the likelihood of a decrease of $10-20 \%){ }^{15}$

\section{Conclusion}

In patients who received a valve replacement for chronic AR, decreased LVEF and large LV dimensions might not affect survival. However, smaller indexed LV systolic and diastolic dimensions and preoperative preserved EF were associated with early restoration of LV function after AVR. Thus, an indexed LV dimension evaluated along with symptoms and LVEF might help predict the postoperative clinical course and prognosis for patients with chronic AR.

\section{References}

1. Carabello BA. Aortic regurgitation: A lesion with similarities to both aortic stenosis and mitral regurgitation. Circulation 1990; 82: $1051-1053$.

2. Ishii K, Hirota Y, Suwa M, Kita Y, Onaka H, Kawamura K. Natural history and left ventricular response in chronic aortic regurgitation. Am J Cardiol 1996; 78: 357-361.

3. Ross J Jr. Afterload mismatch in aortic and mitral valve disease: Implications for surgical therapy. J Am Coll Cardiol 1985; 5: 811 826.

4. Ferrari R, Ceconi C, Campo G, Cangiano E, Cavazza C, Secchiero P, et al. Mechanisms of Remodelling. Circ J 2009; 73: 1973-1982.

5. American College of Cardiology; American Heart Association Task Force on Practice Guidelines (Writing Committee to revise the 1998 guidelines for the management of patients with valvular heart disease); Society of Cardiovascular Anesthesiologists, Bonow RO, Carabello BA, Chatterjee K, de Leon AC Jr, Faxon DP, Freed MD, et al. ACC/AHA 2006 guidelines for the management of patients with valvular heart disease: A report of the American College of Cardiology/American Heart Association Task Force on Practice Guidelines (writing Committee to revise the 1998 guidelines for the management of patients with valvular heart disease) developed in collaboration with the Society of Cardiovascular Anesthesiologists endorsed by the Society for Cardiovascular Angiography and Interventions and the Society of Thoracic Surgeons. J Am Coll Cardiol 2006; 48: e1-e148.

6. Jin XY, Pepper JR, Gibson DG, Yacoub MH. Early changes in the time course of myocardial contraction after correcting aortic regurgitation. Ann Thorac Surg 1999; 67: 139-145.

7. Tornos MP, Olona M, Permanyer-Miralda G, Herrejon MP, Camprecios M, Evangelista A, et al. Clinical outcome of severe asymptomatic chronic aortic regurgitation: A long-term prospective follow-up study. Am Heart J 1995; 130: 333-339.

8. Chaliki HP, Mohty D, Avierinos JF, Scott CG, Schaff HV, Tajik 
AJ, et al. Outcomes after aortic valve replacement in patients with severe aortic regurgitation and markedly reduced left ventricular function. Circulation 2002; 106: 2687-2693.

9. Klodas E, Enriquez-Sarano M, Tajik AJ, Mullany CJ, Bailey KR, Seward JB. Aortic regurgitation complicated by extreme left ventricular dilation: Long-term outcome after surgical correction. J Am Coll Cardiol 1996; 27: 670-677.

10. Bonow RO, Lakatos E, Maron BJ, Epstein SE. Serial long-term assessment of the natural history of asymptomatic patients with chronic aortic regurgitation and normal left ventricular systolic function. Circulation 1991; 84: 1625-1635.

11. Sambola A, Tornos P, Ferreira-Gonzalez I, Evangelista A. Prognostic value of preoperative indexed end-systolic left ventricle diameter in the outcome after surgery in patients with chronic aortic regurgitation. Am Heart J 2008; 155: 1114-1120.

12. Brown ML, Schaff HV, Suri RM, Zhuo L, Sundt TM, Dearani JA, et al. Indexed left ventricular dimensions best predict survival after aortic valve replacement in patients with aortic valve regurgitation. Ann Thorac Surg 2009; 87: 1170-1176.

13. Daimon M, Watanabe H, Abe Y, Hirata K, Hozumi T, Ishii K, et al. Normal values of echocardiographic parameters in relation to age in a healthy Japanese population. Circ J 2008; 72: 1859-1866.
14. Bhudia SK, McCarthy PM, Kumpati GS, Helou J, Hoercher KJ, Rajeswaran J, et al. Improved outcomes after aortic valve surgery for chronic aortic regurgitation with severe left ventricular dysfunction. J Am Coll Cardiol 2007; 49: 1465-1471.

15. Boucher CA, Bingham JB, Osbakken MD. Early changes in left ventricular size and function after correction of left ventricular volume overload. Am J Cardiol 1981; 47: 991-1004.

16. Kang HJ, Kim YJ, Sohn DW, Oh BH, Lee MM, Park YB, et al. Prediction of postoperative left ventricular systolic function with Doppler-derived $\mathrm{dP} / \mathrm{dt}$ in patients with chronic aortic regurgitation. $J$ Am Soc Echocardiogr 2003; 16: 1111-1115.

17. Hirooka K, Yasumura Y, Tsujita Y, Hanatani A, Nakatani S, Hori $\mathrm{M}$, et al. Enhanced method for predicting left ventricular reverse remodeling after surgical repair of aortic regurgitation: Application of ultrasonic tissue characterization. J Am Soc Echocardiogr 2002; 15: $695-701$.

18. Carabello BA. Is it ever too late to operate on the patient with valvular heart disease? J Am Coll Cardiol 2004; 44: 376-383.

19. Fioretti P, Roelandt J, Sclavo M. Postoperative regression of left ventricular dimensions in aortic insufficiency: A long-term echocardiographic study. J Am Coll Cardiol 1985; 5: 856-861. 